\title{
Clinical Profile of Iridocorneal Endothelial Syndrome at a Tertiary Care Eye Centre
}

\author{
Bibhuti Narayanan ${ }^{1}$, Narayanan Balakrishnan ${ }^{2}$, Praveena V. ${ }^{3}$, Vishnu S. ${ }^{4}$
}

\author{
${ }^{1}$ Department of Ophthalmology, Sankara Nethralya Medical Research Foundation, Chennai, \\ Tamilnadu, India. ${ }^{2}$ Department of Ophthalmology, Sri Ramachandra Institute of Higher \\ Education and Research, Chennai, Tamilnadu, India. ${ }^{3}$ Department of Ophthalmology, Sri \\ Ramachandra Institute of Higher Education and Research, Chennai, Tamilnadu, India. \\ ${ }^{4}$ Department of Ophthalmology, Sri Ramachandra Institute of Higher Education and \\ Research, Chennai, Tamilnadu, India.
}

\section{ABSTRACT}

\section{BACKGROUND}

Irido-Corneal Endothelial (ICE) syndrome is an uncommon clinical entity which includes corneal endothelial damage, obstruction of anterior chamber angle, iris atrophy, and polycoria. ICE syndrome is a spectrum of clinical entities namely Progressive Iris Atrophy (PIA), Cogan Reese Syndrome (CRS), and Chandler Syndrome (CS). It is considered to be sporadic, usually unilateral and diagnosed in young adults, females and occasionally in children. Corneal decompensation and glaucoma are the commonest causes of vision loss in patients with ICE syndrome. Medical management of this condition is usually with topical antiglaucoma medication. In most cases medical therapy fails and requires Glaucoma Filtration surgery. Glaucoma drainage devices are the mainstay of treatment and many patients require keratoplasty. We wanted to study the clinical profile of ICE syndrome patients, its association with glaucoma in Indian population and compare the findings with those of previous studies.

\section{METHODS}

In this retrospective study, data of 50 consecutive patients having ICE syndrome at a tertiary eye care centre was collected.

\section{RESULTS}

Out of 50 patients, 28 (56\%) were females, aged 7 to 65 years (median - 40.72 years). 49 patients $(98 \%)$ had unilateral presentation, and one had bilateral involvement (2\%). Progressive Iris Atrophy (PIA) was seen in 20 (40\%), Chandlers Syndrome (CS) in $20(40 \%)$ and 10 eyes (20\%) had Cogan-Reese Syndrome (CRS). 46 (92\%) eyes had glaucoma. Mean Intra-Ocular Pressure (IOP) was $23.70 \pm 11.7 \mathrm{~mm}$ of $\mathrm{Hg}$. Mean AGM used was $2.44 \pm 1.3$. $44(88 \%)$ eyes were diagnosed to have glaucoma at presentation, 1 (2\%) eye was diagnosed with glaucoma during follow up. 20 (40\%) eyes were managed medically for IOP control. 8 (16\%) eyes had undergone previous glaucoma surgery. 20 (40\%) eyes underwent glaucoma surgery for IOP control. 15 (30\%) eyes had Trabeculectomy with Mitomycin C, 5 (10\%) had Ahmed glaucoma valve. $4(8 \%)$ eyes underwent diode cyclophotocoagulation. $6(12 \%)$ eyes required more than 1 surgery for IOP control. 6 (12\%) eyes underwent penetrating keratoplasty (PK) for corneal oedema. Mean period of follow up was $8.4 \pm 6.4$ months. There were no cases of sight threatening complications like suprachoroidal haemorrhage, blindness or endophthalmitis.

\section{CONCLUSIONS}

Glaucoma is strongly associated with ICE syndrome. ICE patients may require more than 1 surgery for IOP control. They are commonly associated with corneal complications which may require penetrating keratoplasty.

\section{KEY WORDS}

Iridocorneal Endothelial Syndrome, Glaucoma, Filtration Surgery
Corresponding Author: Narayanan Balakrishnan, I/17 Plot No. 373, $11^{\text {th }}$ Street, Anna Nagar, East Chennai-600102, Tamilnadu, India.

E-mail: narayanbalakrishnan@hotmail.com

DOI: $10.14260 /$ jemds/2020/667

How to Cite This Article:

Narayanan B, Balakrishnan N, Praveena V, et al. Clinical profile of iridocorneal endothelial syndrome at a tertiary care eye centre. J Evolution Med Dent Sci 2020;9(41):3045-3049, DOI: $10.14260 /$ jemds/2020/667

Submission 11-06-2020,

Peer Review 13-08-2020,

Acceptance 20-08-2020,

Published 12-10-2020.

Copyright (C) 2020 JEMDS. This is an open access article distributed under Creative Commons Attribution License [Attribution 4.0 International (CC BY 4.0)] 


\section{BACKGROUND}

Irido-Corneal Endothelial (ICE) syndrome is an uncommon clinical entity which includes corneal endothelial damage, obstruction of anterior chamber angle, iris atrophy and polycoria. ${ }^{1}$ these proliferative and structural abnormalities are the hallmark of this disease. Corneal decompensation and glaucoma are the commonest causes of vision loss in patients with ICE syndrome. ${ }^{2}$

The aetiology of ICE syndrome is still largely unknown. Inflammation at the level of corneal endothelium due to viral aetiology like Herpes simplex or Epstein-Barr virus and a uveitic pathology have been described earlier.3,4 High percentages of HSV DNA has been found in the endothelial cells of ICE patients compared to controls. ${ }^{4}$

It is considered to be sporadic, usually unilateral and diagnosed in young adults, females and occasionally in children ${ }^{5}$. Syndrome is typically presented with diminution of vision in the morning and change in the shape and position of pupil.

The "membrane theory" was used in 1978 by Campbell to explain the pathogenesis of ICE syndrome. They hypothesized that corneal endothelial cell can proliferate and may develop structural defects with the ability to migrate causing the changes noted in ICE syndrome. ${ }^{6}$

Similar to Fuchs' dystrophy, the slit lamp picture in ICE shows "Hammer silver" or "Beaten bronze" appearance of the endothelium. Also, Iris changes (such as heterochromia, ectropion uveae, corectopia, whole formation, and iris atrophy) are seen on slit-lamp examination. Gonioscopic examination may show high PAS extending above Schwalbe line, which is pathognomonic for ICE syndrome. ${ }^{7}$ Slit lamp examination, gonioscopy, tonometry, specular microscopy and confocal microscopy form an essential part of the examination framework used to diagnose the subclinical variants of ICE syndrome that may manifest itself asymptomatically in the unaffected eye. ${ }^{8}$

Progressive essential iris atrophy, Cogan-Reese syndrome, and Chandler syndrome form the extending spectrum of ICE syndrome and are often considered the three subtypes of the disease. ${ }^{8,} 9$ these three subtypes are difficult to demarcate clinically, and diagnostic differentiation is dependent on the extent of iris defects. Chandler syndrome is the commonest subtype. ${ }^{10}$

Progressive iris atrophy is signified by two types of iris holes - stretch holes, resulting from thinning of the iris on the other side of the direction of pupillary distortion, and melting holes, in which the iris tissue vanishes without tissue ischemia. ${ }^{8}$ Polycoria, corectopia, iris heterochromia and ectropion uveae can occur. ${ }^{7}$ IOP elevation is often due to angle endothelialization and peripheral anterior synechiae formation which results in angle closure, that can be picked up by gonioscopy. Ectropion uveae and heterochromia of iris have also been noted. ${ }^{3}$

Cogan-Reese syndrome is characterized by different degrees of iris atrophy, multiple pedunculated iris nodules, surrounded by iris stroma with a matted appearance due to the absence of normal iris crypts. Fine yellow nodules on the iris surface appear late in the disease process and they often change colour and increase in number. ${ }^{8,11}$
Chandler's syndrome shows minimal iris involvement and is the most common subtype. The corneal endothelium has a characteristic hammered silver appearance, corneal oedema and bullae are the other features that manifest in the cornea. Full thickness iris holes with iris atrophy maybe present. ${ }^{12}$

The major vision threatening sequelae is corneal oedema and glaucoma. Glaucoma is strongly associated with ICE syndrome.2,13

Mechanism of glaucoma is that altered corneal endothelial cells have migratory characteristics and hence it moves posteriorly onto the trabecular meshwork and peripheral iris. Peripheral anterior synechiae results from contraction of this tissue within the angle, causing secondary angle closure glaucoma.

The corneal oedema in ICE syndrome occurs as a result of both elevated intraocular pressure (IOP) from secondary angle-closure glaucoma, and from subnormal pump function from the altered endothelium. ${ }^{14}$ Corneal oedema is seen more commonly in patients with Chandler syndrome, though this can be found in any of the three clinical variants.

The main aim of treatment in the ICE syndrome is the prevention and management of the vision threatening complications like corneal oedema and secondary angle closure glaucoma. With the aim to reduce the IOP which may aid in the improvement of corneal oedema, topical antiglaucoma medications have become the mainstay of treatment. ${ }^{8}$ In literature medical management has been associated with high rates of failure ( 60 to $88 \%$ ) and the need for surgery in most cases have been reported.2,15 Glaucoma filtration surgery in ICE syndrome related glaucoma has shown high rates of failure. ${ }^{2}$ Abnormal endothelial membrane growth over the ostium and trabecular meshwork has been hypothesized to be one of the primary causes of surgical failure resulting in poor intraocular pressure control postsurgery. ${ }^{11}$ The need for GDD such as Barvaeldt, Ahmed glaucoma valve, Molteno with anti-glaucoma medications have offered an effective alternative to trabeculectomy and have been able to lower IOP in most cases. However failure of GDI can occur as there might be pre-existing sub conjunctival fibrosis due to previous trabeculectomies, blockage of the tube due to presence of an ICE membrane that may have recurred and corneal decompensation. ${ }^{16}$ If surgical success is not obtained with trabeculectomy or drainage devices, Cyclodestructive procedures such as cyclophotocoagulation may be needed. Intraocular pressure control in ICE syndrome patients may be difficult because they are younger and have pronounced fibrotic responses that may result in failure of filtration surgery. ${ }^{2}$

Penetrating keratoplasty is required along with glaucoma filtration surgery in advanced cases of ICE syndrome with severe corneal oedema to improve vision, reduce pain, provide a clear media to visualize and quantify the optic nerve head and visual field changes in patients with associated glaucoma.17,18 Descemet stripping endothelial keratoplasty (DSEK) has also provided good short-term visual outcomes in eyes with ICE syndrome with rapid recovery and minimal astigmatism. ${ }^{19}$ There are a few case series reported so far on ICE syndrome. Shields ET al $^{1}$ reported 82 patients of ICE syndrome in Caucasian population, Teekhasaenee and Reech $^{20}$, in 60 eyes in Thai population. Recently in 2000 Chandran et $\mathrm{al}^{13}$ has done study on 203 eyes in Indian population. 
We wanted to study the clinical profile of ICE syndrome patients, its association with glaucoma in Indian population and compare the findings with those of previous studies.

\section{METHODS}

All patients diagnosed with ICE syndrome as mentioned in our inclusion criteria diagnosed between the October 2016October 2019. A total of 50 patients were included in our study after ruling out exclusion criteria and the data was retrospectively analysed.

Data pertaining to age, gender, clinical spectrum of disease, laterality, Visual acuity, corneal and iris findings, anterior chamber (AC) depth, gonioscopic findings, reaction in AC, association with glaucoma, medical and surgical management was collected, number of AGMs used, duration of follow up collected.

Complete slit lamp biomicroscopy, applanation tonometry, four mirror gonioscopy, dilated fundoscopy were performed to determine corneal changes, iris findings, AC reaction, PAS, intraocular pressure and Disc changes. In some patients, presence of severe corneal oedema obviated the performance of specular microscopy and PAS assessment. For diagnosing glaucoma Humphrey Visual field test was done in presence of clear cornea and sufficient vision.

\section{Inclusion Criteria}

ICE syndrome is defined on the basis of characteristic findings of beaten-bronze or hammered-silver appearance of endothelium, iris atrophy with hole and iris nodules with or without PAS.

- IOP $>21 \mathrm{~mm}$ of $\mathrm{Hg}$ or $</=21 \mathrm{~mm}$ of $\mathrm{Hg}$ with AGM/prior Glaucoma filtration surgery (GFS) with optic nerve head changes characteristic of glaucoma was a criterion to diagnose glaucoma in the presenting patients.

- Secondary ocular hypertension (OHT) was also included as glaucoma patients in our study.

- Patients were classified as CS, PIA and CRS on the basis of normal or mild iris atrophy with predominant corneal involvement, iris atrophy with whole formation, iris nodules respectively.

- Cornea was graded as clear or oedematous.

- PAS was graded as mild $(<90)$, moderate (90 to 180$)$, severe $(>180-270)$ and extensive $(>270)$.

\section{Exclusion Criteria}

- Axenfeld-Reiger syndrome

- Fuchs endothelial dystrophy.

- Uveitis, traumatic angle closure.

- Other primary and secondary angle closure glaucoma were excluded.

\section{Statistical Analysis}

We undertook a retrospective analysis of all study patients and obtained data pertaining to clinical characteristics. Since the data was non-parametric, we used chi squared test and student $\mathrm{T}$ test to analyse the data. ANOVA was also performed to look for variance. Analyses were conducted using StataCorp 97: Stata Statistical Software Release 5 (Stata Corporation, USA) and StatXact version 2 (Cytel Software Corporation, Cambridge, MA, USA).

\section{RESULTS}

We studied 51 eyes of 50 patients with age range of 7-65 years with median age at presentation was 40.72 years. 28 (56\%) were female and 22 (44\%) were male. ICE syndrome was unilateral in 49 (98\%), of which right was involved in 25 (50\%) and left was involved in 24 (48\%). Mean IOP of involved eye was $23.70 \pm 11.7 \mathrm{~mm}$ of $\mathrm{Hg}$. PIA and CS variant of ICE syndrome were equal in frequency of $20(40 \%)$. CRS was found as least variant of ICE syndrome and was $10(20 \%)$ patients. Out of 51 eyes of study group we had 43 (84.3\%) eyes were phakic and 7 (13.7\%) eyes were pseudophakic. There were no aphakic eye in our study group patients. [Graph1]

$44(88 \%)$ eyes were diagnosed to have glaucoma at presentation, $1(2 \%)$ eye was diagnosed with glaucoma during follow up.

Out of 45 patients who all diagnosed as glaucoma at presentation or during follow up 20 (44.4\%) patients were treated with AGMs and remaining 25 (55.5\%) patients were treated as glaucoma filtration surgery (GFS). [graph 2] Mean no of AGMs used were $2.44 \pm 1.3$. Out of 25 patients treated with GFS, 20 (44.4\%) eyes undergone trabeculectomy and 5 (11.1\%) undergone Ahmed glaucoma valve. 8 (17.7\%) eyes had previous GFS. [Graph 3] Number of eyes had Diode CPC were 4 (8.8\%). $6(13.3 \%)$ eyes were undergone more than 1 surgeries to control the IOP during the follow up. We have found in our study that median age of patients undergone medical and surgical management of glaucoma was 44.53 years (range 7-56) and 46.26 years (range 13-60). (Table 1)

\begin{tabular}{|cc|}
\hline Gender & \\
Male & $22(46 \%)$ \\
Female & $28(56 \%)$ \\
Laterality & \\
OD & $25(50 \%)$ \\
OS & $24(48 \%)$ \\
OU & $1(2 \%)$ \\
Mean IOP (mm of Hg) & $23.70 \pm 11.7$ \\
Mean No. of antiglaucoma medications & $2.44 \pm 1.3$ \\
Lens status of eyes & $43(84.3 \%)$ \\
Phakic & $7(13.7 \%)$ \\
Pseudophakic & 0 \\
Aphakic & $8.4 \pm 6.4$ \\
\hline Follow up period (Months) \\
\hline Clinical Profile of Ice Syndrome Patients \\
\multicolumn{2}{|c}{} \\
\hline
\end{tabular}

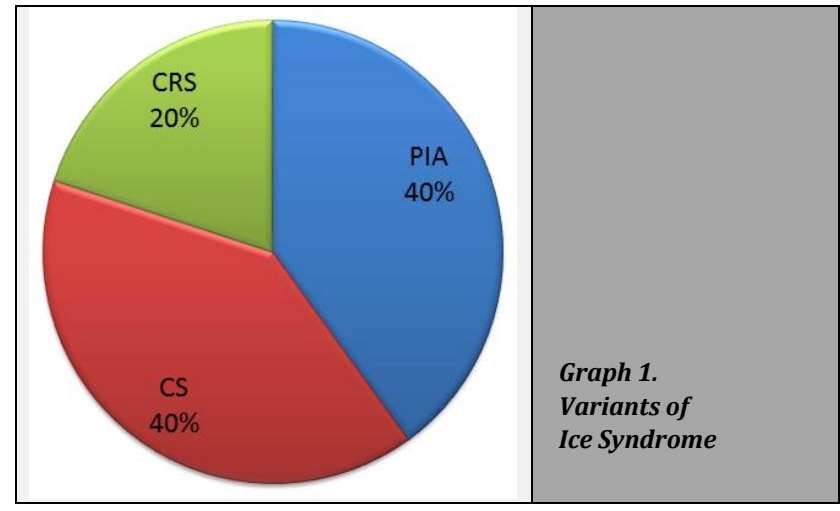



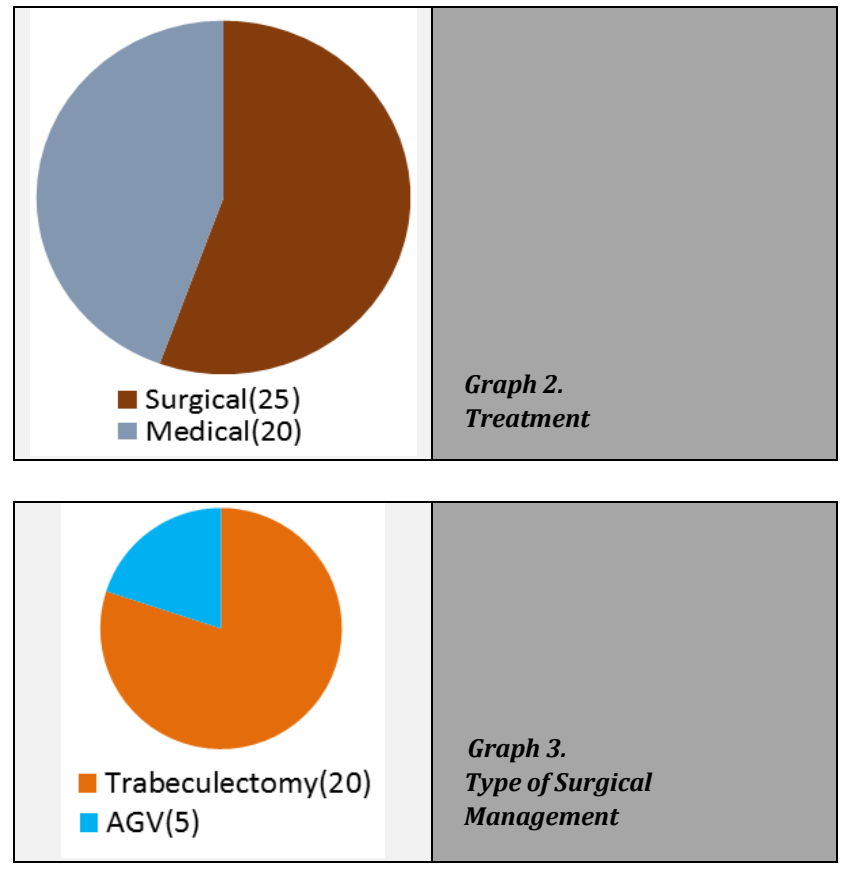

\section{DISCUSSION}

Corneal endothelial cells have been noted to proliferate and grow over the angle of the anterior chamber and iris and this is pathognomonic of ICE syndrome. There are three clinical variants of ICE syndrome. In our study median age of presentation was 40.72. The average age of presentation of ICE syndrome in studies done by Wilson et al, Teekhasaenee et al, Shield et al, Chandran et al were 41.5 years, 43.7 years, 38.6 years and 43 years respectively.110,13,14 Females (56\%) were affected more than males, which is similar to previously published studies, Wilson et al (62.2\%),10 Teekhasaenee et al ${ }^{20}$ (66.7\%), Shield et $\mathrm{al}^{1}$ (68.3\%) and Chandran et $\mathrm{al}^{13}$ (61\%). 17 patients were studied by Hirst et al., all being females, 12 females, 3 males was the gender variants observed in ICE syndrome in a study by Liu et al and 10 females, 2 males were reported to have ICE syndrome by Le et al. 66 ICE patients were studied by Laganowski et al., of which 31 were males $(47 \%)$ and 35 were females (53\%). ${ }^{2}$

In our study PIA and CS variants are of equal frequency $(40 \%)$. CRS $(20 \%)$ was the least common variant of ICE syndrome which was similar to Chandran et al in which PIA (52\%) was the predominant variant followed by CS and the least was CRS.13 Laganowski et al case series also showed the similar frequency ${ }^{2}$. Wilson et al ${ }^{10}$ reported that Cogan-Reese was the commonest in Caucasian population, whereas Teekhasaenee et al reported that Cogan- Reese syndrome was common in the Thai population. ${ }^{14}$ This is suggestive of the existence of an ethnic variation in the presentation of the disease.

ICE syndrome is typically unilateral in presentation. In our study $98 \%$ of the patients were having unilateral involvement, which was similar to previous studies also. Glaucoma is strongly associated with ICE syndrome. The prevalence of glaucoma in our study was $90 \%$. It ranges from $46 \%$ to $82 \%{ }^{1,2}$ In our study $44.4 \%$ of glaucoma patients were managed with antiglaucoma medications. In other published series it ranged from $12-40 \% \cdot 1,2,13,14$
These differences can be possibly explained by the variations in the severity of the disease and the evolution of anti-glaucoma medications over time. Chandan et al studied a case series of 202 patients and found an inter eye variance in the presentation of ICE syndrome. Unilaterality was exhibited in $183(90 \%)$ subjects, and bilaterality in $20(10 \%)$ subjects. Progressive iris atrophy ( $115 ; 52 \%$ eyes), Chandler syndrome (87; 39\% eyes) and Cogan-Reese syndrome $(21 ; 9 \%$ eyes) were found in decreasing order of frequency. 156 eyes (70\%) had glaucoma at initial presentation and the average intraocular pressure in glaucomatous eyes was $24(16,38)$ mmHg. ${ }^{13}$ Glaucoma filtration surgery for IOP control was required in our study was $55.5 \%$ patients.

As glaucoma in ICE syndrome is refractive in nature surgical management is required to control the IOP. In our study $44.4 \%$ eyes undergone trabeculectomy and $11.1 \%$ undergone Ahmed glaucoma valve. $8.8 \%$ of eyes had Diode CPC. $13.3 \%$ eyes were undergone more than 1 surgeries to control the IOP during the follow up. 13.3\% eyes were undergone keratoplasty for corneal decompensation. Studies by Lanzl et al Doe et al have shown moderate success with trabeculectomy as a primary surgery for patients with ICE syndrome ranging between $64-80 \% .^{21,22}$ There aren't too many studies that have analysed surgical outcomes over a longer time frame. Most studies have shown that more than one filtering surgery was required in most patients. These results were comparable to our study. Younger age, ostial block by a recalcitrant membrane and iris tissue are the factors responsible for the failure of filtration surgery. Aggressive bleb fibrosis is an important factor for filtration failure. ${ }^{2}$

\section{CONCLUSIONS}

In our study, ICE syndrome was unilateral, and middle-aged females were more commonly affected. Glaucoma is strongly associated with ICE syndrome. Surgical management is required in majority of the affected patients. ICE patients may require more than 1 surgery for IOP control. They are commonly associated with corneal complications which may require penetrating keratoplasty.

Financial or Other Competing Interests: None.

\section{REFERENCES}

[1] Shields MB. Progressive essential iris atrophy, Chandler's syndrome, and the iris nevus (Cogan-Reese) syndrome: a spectrum of disease. Survey of Ophthalmology 1979; 24(1):3-20.

[2] Laganowski HC, Muir MGK, Hitchings RA. Glaucoma and the iridocorneal endothelial syndrome. Arch Ophthalmol 1992; 110(3):346-50.

[3] Scheie HG, Yanoff M. Iris nevus (Cogan-Reese) syndrome. A cause of unilateral glaucoma. Arch Ophthalmol 1975; 93(10):963-70. 
[4] Alvarado JA, Underwood JL, Green WR, et al. Detection of herpes simplex viral DNA in the iridocorneal endothelial syndrome. Arch Ophthalmol 1994; 112(12):1601-9.

[5] Salim S, Shields MB, Walton D. Iridocorneal endothelial syndrome in a child. J Pediatr Ophthalmol Strabismus 2006; 43(5):308-10.

[6] Sherrard ES, Frangoulis MA, Muir MG, et al. The posterior surface of the cornea in the iridocorneal endothelial syndrome: a specular microscopical study. Trans Ophthalmol Soc U K 1985; 104(Pt 7):766-74.

[7] Glaucoma. Basic and Clinical Science Course (BCSC). American Academy of Ophthalmology 2010-2011:142-4.

[8] Sacchetti M, Mantelli F, Marenco M, et al. Diagnosis and management of iridocorneal endothelial syndrome. Biomed Res Int 2015; 2015:1-9.

[9] Campbell DG, Shields MB, Smith TR. The corneal endothelium and the spectrum of essential iris atrophy. Am J Ophthalmol 1978; 86(3):317-24.

[10] Wilson MC, Shields MB. A comparison of the clinical variations of the iridocorneal endothelial syndrome. Arch Ophthalmol 1989; 107(10):1465-8.

[11] Eagle RC, Font RL, Yanoff $M$, et al. Proliferative endotheliopathy with iris abnormalities. The iridocorneal endothelial syndrome. Arch Ophthalmol 1979; 97(11):2104-11.

[12] Chandler PA. Atrophy of the stroma of the iris, endothelial dystrophy, corneal oedema, and glaucoma. Trans Am Ophthalmol Soc 1955; 53:75-89.

[13] Chandran P, Rao HL, Mandal AK, et al. Glaucoma associated with iridocorneal endothelial syndrome in 203 Indian subjects. PloS One 2017; 12(3):e0171884.
[14] Levy SG, Kirkness CM, Moss J, et al. The histopathology of the iridocorneal-endothelial syndrome. Cornea 1996; 15(1):46-54.

[15] Shields MB, Campbell DG, Simmons RJ. The essential iris atrophies. Am J Ophthalmol 1978;85(6):749-59.

[16] Kim DK, Aslanides IM, Schmidt CM, et al. Long-term outcome of aqueous shunt surgery in ten patients with iridocorneal endothelial syndrome. Ophthalmology 1999; 106(5):1030-4.

[17] Shields MB, McCracken JS, Klintworth GK, et al. Corneal oedema in essential iris atrophy. Ophthalmology 1979; 86(8):1533-50.

[18] Alvim PT, Cohen EJ, Rapuano CJ, et al. Penetrating keratoplasty in iridocorneal endothelial syndrome. Cornea 2001; 20(2):134-40.

[19] Fajgenbaum MA, Hollick EJ. Descemet stripping endothelial keratoplasty in iridocorneal endothelial syndrome: postoperative complications and long-term outcomes. Cornea 2015; 34(10):1252-8.

[20] Teekhasaenee C, Ritch R. Iridocorneal endothelial syndrome in Thai patient: clinical variations. Arch Ophthalmol 2000;118(2):187-92.

[21] Lanzl IM, Wilson RP, Dudley D, et al. Outcome of trabeculectomy with mitomycin-C in the iridocorneal endothelial syndrome. Ophthalmology 2000; 107 (2):2957.

[22] Doe EA, Budenz DL, Gedde SJ, et al. Long-term surgical outcomes of patients with glaucoma secondary to the iridocorneal endothelial syndrome. Ophthalmology 2001; 108(10):1789-95. 